Overcoming interaction barriers in large public displays using personal devices Peer-reviewed author version

Cheung, Victor; Watson, Diane; VERMEULEN, Jo; Hancock, Mark \& Scott D., Stacey (2014) Overcoming interaction barriers in large public displays using personal devices. In: ITS '14 Proceedings of the Ninth ACM International Conference on Interactive Tabletops and Surfaces, p. 375-380.

DOI: $10.1145 / 2669485.2669549$

Handle: http://hdl.handle.net/1942/17835 


\section{Overcoming Interaction Barriers in Large Public Displays Using Personal Devices}

\author{
Victor Cheung \\ victor.cheung@uwaterloo.ca \\ Systems Design Engineering \\ University of Waterloo \\ Waterloo, Ontario, Canada \\ Diane Watson \\ diane.watson@uwaterloo.ca \\ Computer Science \\ University of Waterloo \\ Waterloo, ON, Canada

\section{Jo Vermeulen} \\ jo.vermeulen@hasselt.be \\ Hasselt University - tUL - iMinds \\ Expertise Centre for Digital Media \\ Diepenbeek, Belgium
}

Permission to make digital or hard copies of part or all of this work for personal or classroom use is granted without fee provided that copies are not made or distributed for profit or commercial advantage and that copies bear this notice and the full citation on the first page. Copyrights for third-party components of this work must be honored. For all other uses, contact the Owner/Author.

Copyright is held by the owner/author(s).

ITS '14, Nov 16-19 2014, Dresden, Germany ACM $978-1-4503-2587-5 / 14 / 11$

http://dx.doi.org/10.1145/2669485.2669549

\section{Mark Hancock}

cock@uwaterloo.ca

Management Sciences

ersity of Waterloo

Stacey D. Scott

stacey.scott@uwaterloo.ca

Systems Design Engineering

University of Waterloo

Waterloo, Ontario, Canada

\begin{abstract}
This work presents a design space in which personal devices are used as a means to facilitate "socially safe", ad-hoc interaction with large public displays. Unlike most existing work that focuses on facilitating content placement and transfer, this approach aims at minimizing the effort required to initiate, sustain, and withdraw from interaction with a large public display, and to communicate these capabilities to passersby. We identify barriers hindering this process, and offer advice on overcoming them based on existing work and our own experiences with these displays. We illustrate how this design concept can be applied, and motivate applications in other domains.
\end{abstract}

\section{Introduction}

Large interactive displays are often installed in public spaces (e.g., museums, airports, storefronts) to provide a more engaging experience. However, studies have shown that they are highly underutilized, largely due to social inhibition (e.g., fear of looking foolish) $[4,21]$ and uncertainty of possible interactions $[12,18]$. Similar to gestural interfaces, interaction with large public displays typically suffers from a lack of visibility ("what functionality is supported?") and affordances ("what actions are possible?") [17]. Moreover, due to constraints such as cost, lighting, and space, common interaction mechanisms such as touch, sound, and body 
movements might be inhibited or only available to a small audience. Meanwhile, personal devices, such as smartphones and tablets, have become a widespread means for interacting with digital content. Besides being technologically capable of supporting various forms of interaction (e.g., touching, tilting, or shaking), these devices are familiar and allow personalization, and thus enjoy higher adoption [7].

In this paper, we explore a design space where personal devices are used as an alternative entry point to initiate and sustain interaction with large public displays. By taking advantage of people's familiarity and lower social inhibition with their own personal devices, and by improving the public display's ease of use and intelligibility [2], this form of interaction may instill confidence in passersby. It may also allow for a broader range of audience by, for example, helping museum patrons store information for later use, enabling pedestrians to overcome social inhibition with storefront displays, or assisting people with physical disabilities to engage with large displays.

Existing multi-surface research involving large and small displays largely focuses on content placement and transfer between devices. Instead, we focus on exploring the use of personal devices to encourage large public display use, thereby benefiting a broader audience. Our three main contributions are: 1 ) synthesizing design challenges of large public display use as a set of usage barriers, 2) offering design advice to overcome these barriers, and 3) describing a representative application for further development.

\section{Classifying Existing Cross Device Work}

While touch devices are often praised for supporting direct interaction-when the control and display space are super-imposed-prior research has explored using one device to indirectly control another $[4-6,8,11]$, for example, to facilitate content transfer between large displays and personal devices $[8,13,15]$. We extend the idea of direct vs. indirect interaction by situating existing work in a cross-device control-display space:

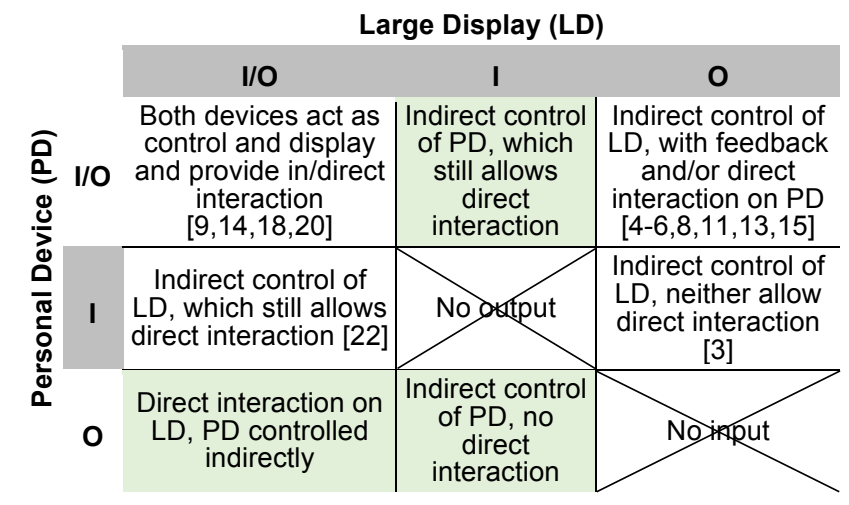

Table 1. Cross-device control display space. Both the large display (LD) and the personal device (PD) can be used as input only (I), output only (O), or as both input and output (I/O).

Research thus far has focused on using large displays for output (column 3), with personal devices used as input, either with (row 1) or without (row 2) feedback on the devices. The large display is also sometimes interactive (column 1). However, much of this research space remains unexplored, such as the use of personal devices as passive displays (row 3, column 1) or the use of large displays to indirectly control personal devices (column 2), e.g., to activate private information. 


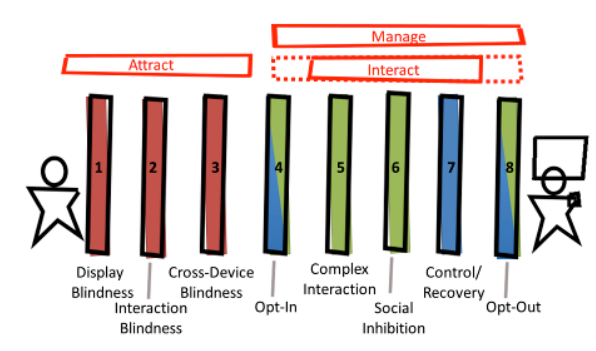

Figure 1. Partial ordering of cross-device barriers.

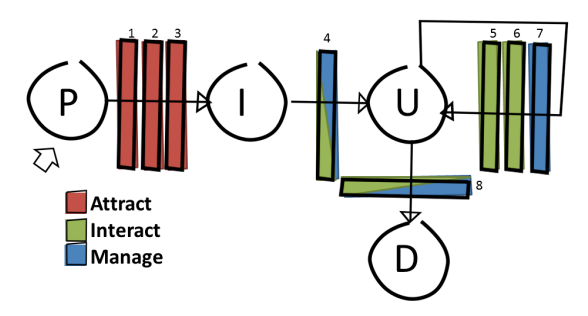

Figure 2. Diagram showing various states of interaction and barriers encountered. (P:Passing, I:Interested, U:Using, D:Done)
In this work, we consider the large display as a potential attractor (columns $1 \& 3$ ), and use the familiarity of input on personal devices (row $1 \& 2$ ) to promote engagement. Yet, many barriers remain to entice a seamless transition from single device use to cross-device interaction.

\section{Cross-device Barriers in Public Spaces}

Even with a similar hardware setup, the interaction style between large displays and personal devices in a public setting differs from those in non-public settings like classrooms and workspaces. We build upon Echtler et al.'s [9] notion of "casual interaction", where the joining step is kept minimal and no modification of the personal devices is required, and highlight the requirements of approach-and-use and graceful withdrawal [4]. The interaction process is comprised of personal devices (capturing unique traits such as privacy and novel interaction mechanisms [1]), and a large display (which is used as a shared display, and a direct input alternative, if supported).

This style of interaction poses several usage barriers for engaging passersby. We list these barriers in Figure 1 in partial order of their appearance and cluster them conceptually into attract, interact, and manage.

Attract: Before any interaction, the large display must first attract passersby's attention and interest. They first must notice the display (hindered by display blindness), be aware that one can interact with it (hindered by interaction blindness) and recognize they can use their personal device as a means of interaction (hindered by cross-device blindness).

Interact: During interaction, it must be easy to understand and make use of the large display and personal device (hindered by complex interaction). Users must also feel comfortable with the interaction (hindered by social inhibition). This stage may also involve opt-in and opt-out mechanisms for users to engage in or withdraw from using the system [10].

Manage: The connection between the large display and personal device must be maintained and communicated. Opting-in (connection), opting-out (explicit disconnection), or leaving the vicinity (implicit disconnection), must be handled appropriately, and interruptions (e.g., phone call) must be recovered from. It may be frustrating if these control/recovery actions are confusing or non-existent.

The interaction process can be modelled as a series of states (Figure 2) and ideally, a passerby will proceed through each state and engage with the system. One starts by passing $(P)$ the display, and if attracted, they will become interested/observe the display (I). If desired, they will opt-in, then use the system (U) Finally, they will opt-out and be done (D). At any transition, they may encounter a barrier and become disinterested or leave. Figure 2 details movement from state to state and when the barriers in attract, interact, and manage may be encountered. Next, we offer design advice to overcome these barriers.

\section{Overcoming Attract Barriers}

React to Presence (A1): A way to overcome display blindness is to react subtly to the presence of passersby. If tracking capabilities are available, the display can react based on presence or movements in front of the display, e.g., by dynamically changing the content to increase audience attention towards the large display $[16,23]$ 
Provide Instructions (A2): Providing signs or instruction labels near the large display [12] can indicate that the display is interactive. Next to static instructions, the display can show animations or videos illustrating how it can be used (e.g., a self-revealing help system [24]). Based on the purpose of the context (e.g., one-time or frequent use), a combination of static instructions and animated demonstration can be included in the interface to promote learning and recall [19].

Provide Signifiers (A3): Exposing technology (e.g., sensors) is another way to convey that the display is interactive. Other recognizable technology can be used with the display. For example, a QR code can by itself be an affordance that informs those who have encountered these codes before about the potential for action [14] (although it does not describe what can be done). Once someone begins interacting with the display, the honeypot effect [4] can draw others in.

\section{Overcoming Interact Barriers}

Support Immediate Interaction (I1): Connections should be possible with minimal interaction (e.g., taking a photo instead of installing an app, a shorter URL link). Redundant means of connection can be used to reach more users with different devices [8]. Also, the system should not require extensive training for use.

Convey Possible Input Modalities (I2): Personal devices typically provide several input modalities, such as touch, text and accelerometer data [1], and may come in different sizes and shapes. The large display itself may also support touch, full body gestures, or the ability to locate people. Care should be taken to clearly convey the action possibilities, and how people can interact with the system. Similar to connecting to the display, we argue for a graceful degradation approach, where multiple ways of interacting are possible (e.g., use of touch buttons or gestures when accelerometer data is unavailable on the personal device).

Provide Appropriate Feedback (I3): It is essential to provide feedback during display interaction, device connection, or connection failures. We see feedback as a key strategy to overcome social inhibition, as confusion over system state or what interaction is possible is a common cause of social embarrassment. Reducing the fear of failure can reduce social inhibition.

Offer Hints/Exploration (I4): One way to convey how to interact with the system is to provide time to explore the interaction mechanisms. For example, Google's Super Sports Sync game (http://g.co/super) allows people to try out the interaction after they are connected but before the game starts. This teaches people the impact of their actions. Synchronized feedback between the large display and personal device can also be used to make the connection more visible.

\section{Overcoming Manage Barriers}

Minimize Necessary Bandwidth (M1): To support multiple devices connecting to the same display, care should be taken to reduce messages sent between each device and the display. For example, device orientation information can be sent at a reasonable interval and interpolated in-between.

Conduct Opt-In/Opt-Out (M2): Opting in to using a personal device should always be explicit. The system should not start a download or automatically open an app as this can break trust [10]. If personal data will be exposed (e.g., social networking data), it should be very clear when the system is using or purging the data (such as when someone leaves). 


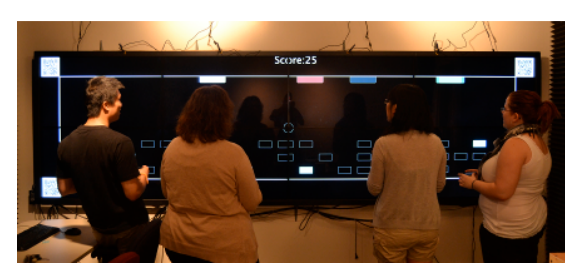

Figure 3. Multiplayer Breakout. Each player controls a paddle via their mobile device connected to the large display.

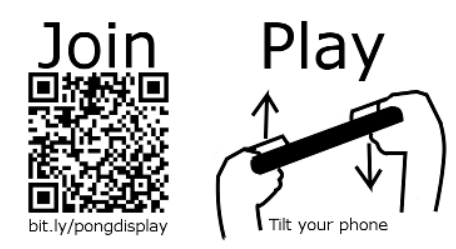

Figure 4. Simple signage to be posted near the public display.
Supporting an opt-in approach can also address issues of accidental interaction by passive observers. For instance, a problem of ubiquitous systems in public spaces is that they often utilize implicit input, where someone walking by may inadvertently trigger the display [2]. The personal device could provide explicit opt-in by, for instance, outputting a code or sequence of flashing lights (detected by the large display's sensors) for authentication. Then, the large display would only accept input from those who have opted-in.

Support Recovery \& Control (M3): Some people may not follow "proper" disconnection procedures when leaving the display. The system should allow graceful disconnection after a period of inactivity or distance from the display. Reconnection should also be possible if failure or interruption (e.g., a phone call) occurs. Sensible defaults should exist for both the large display and personal devices.

\section{Illustrative Prototype}

To illustrate how the above design advice can be applied, we are developing a prototype where a large public display is used as the main attractor to a multiplayer version of the classic arcade game,

Breakout. Each player controls a paddle that moves left and right to intercept the ball via their personal device. Points are earned when the ball hits a brick, and lost when the ball is not intercepted and goes off-screen.

To overcome Attract Barriers, simple signage (A2), as shown in Figure 4, is used to notify users that interaction is possible. Connection tokens such as QR code and URL link are used due to their familiarity to mobile users (A3). These tokens can be animated when a passerby is in proximity to draw their attention (A1).
To overcome Interact Barriers, upon taking a picture of the QR code, or entering the URL link into their web browser, players are immediately connected to the game, without any extra download or installation (I1). Players are randomly assigned a paddle color, which is displayed on their personal device (I3). Control mechanisms (e.g. tilt, touch-buttons) are displayed according to the device capabilities and appropriately mapped to paddle's movements (I2). Information about the player's connection status (error, connected, or disconnected) pops up on the personal device upon changes (I3). The game provides a countdown when the first person connects before starting the game to enable exploration of the interaction mechanism (14).

To overcome Manage Barriers, interruptions (e.g., the phone goes to sleep, incoming calls) are handled by freezing the paddle in place on the large display until the player returns to the web browser on their personal device (M3). Players can leave or enter the game at any point; the game pauses when there is no player (M3). Inactive players will eventually timeout and be removed from play (M2). Device orientation messages are only sent at short intervals $(100 \mathrm{~ms})$ to reduce the required bandwidth (M1).

We are planning to incorporate more interactive game elements into both the large display and mobile device, and investigate how users are enticed to interact with large public displays using this cross-device interaction.

\section{Conclusion and Future Work}

In this paper, we classify existing literature using our cross-device control-display space. We then describe a design framework that identifies barriers to crossdevice interaction, and suggest methods of addressing 
these barriers. We demonstrate our design framework through an example of the multiplayer Breakout game on a public wall display. In the future, we intend to evaluate different techniques for overcoming each barrier to interaction, and explore the use of this framework in other settings.

\section{References}

[1] Ballagas, R., Borchers, J. et al. (2006). The smart phone: a ubiquitous input device. IEEE Pervasive Computing 5(1), 70-77.

[2] Bellotti, V. and Edwards, K. (2001). Intelligibility and accountability: human considerations in context-aware systems. Hum.-Comput. Interact. 16(2), 193-212.

[3] Boring, S., Jurmu, M., \& Butz, A. Scroll, tilt or move it: using mobile phones to continuously control pointers on large public displays. Proc. OZCHI '09, 161-168.

[4] Brignull, H. and Rogers, Y. (2003) Enticing people to interact with large public displays in public spaces. INTERACT, 17-24.

[5] Cao, X., Massimi, M. and Balakrishnan, R. (2008). Flashlight jigsaw: an exploratory study of an ad-hoc multiplayer game on public displays. CSCW '08, 77-86.

[6] Carter, S., Churchill, E. et al. (2004). Digital graffiti: public annotation of multimedia content. Ext. Abs. CHI '04, 1207-1210.

[7] Clinch, S. (2013). Smartphones and pervasive public displays. IEEE Pervasive Computing, 12(1), 92-95.

[8] Dearman, D. \& Truong, K.N. (2009). BlueTone: a framework for interacting with public displays using dual-tone multi-frequency through bluetooth. Ubicomp '09, 97-100.

[9] Echtler, F, Nestler, S. et al. (2009). Supporting casual interactions between board games on public tabletop displays and mobile devices. Personal Ubiquitous Computing, 13(8), 609-617.

[10] Greenberg, S., Boring, S. et al. (2014). Dark patterns in proxemic interactions: a critical perspective. DIS '14, 523-532.

[11] Izadi, S., Brignull, H. et al. (2003). Dynamo: a public interactive surface supporting the cooperative sharing and exchange of media. UIST '03, 159-168.
[12] Ju, W. and Sirkin, D. (2010). Animated objects: how physical motion encourage public interaction. Persuasive Technology, Springer-Verlag: 40-51.

[13] Kaviani, N., Finke, M. et al. (2009). What goes where?: designing interactive large public display applications for mobile device interaction. ICIMCS '09, 129-138.

[14] Luojus, P., Koskela, J. et al. (2013). Wordster: collaborative versus competitive gaming using interactive public displays and mobile phones. PerDis '13, 109-114.

[15] Müller, J., Jentsch, M. et al. (2008). Exploring factors that influence the combined use of mobile devices and public displays for pedestrian navigation. NordiCHI '08 308-317.

[16] Müller, J., Wilmsmann, D. et al. (2009). Display blindness: the effect of expectations on attention towards digital signage. Pervasive Computing, Springer-Verlag:1-8. [17] Norman, D.A. (2002). The Design of Everyday Things. Basic Books, Inc.

[18] Ojala, T., Kostakos, V. et al. (2012). Multipurpose interactive public displays in the wild: three years later. Computer, 45(5), 42-49.

[19] Palmiter, S.L., Elkerton, J. and Baggett, P. (1991) Animated demonstrations vs written instructions for

learning procedural tasks: a preliminary investigation. Int'l. Journal of Man-Machine Studies, 34(5), 687-701.

[20] Peltonen, P., Salovaara, A. et al. (2007). Extending large-scale event participation with user-created mobile media on a public display. MUM '07, 131-138.

[21] Reeves, S., Benford, S. et al. (2005). Designing the spectator experience. CHI '05, 741-750.

[22] Song, P., Goh, W.B. et al. (2011). WYSIWYF: exploring and annotating volume data with a tangible handheld device. Proc. CHI '11, 1333-1342.

[23] Wang, M., Boring, S. \& Greenberg, S. (2012). Proxemic peddler: a public advertising display that captures \& preserves the attention of a passerby. PerDis $12,(3) 1-6$.

[24] Vogel, D. and Balakrishnan, R. (2004). Interactive public ambient displays: Transitioning from implicit to explicit, public to personal, interaction with multiple users. UIST '04, 137-146. 